\title{
Growth characteristics of diabetic rat ectoplacental cones in vivo and in vitro
}

\author{
S. Caluwaerts, R. Pijnenborg, C. Luyten, F. A.Van Assche \\ Department of Obstetrics and Gynaecology, Katholieke Universiteit Leuven, Leuven, Belgium
}

\section{Abstract}

Aims/hypothesis. To investigate the outgrowth of the ectoplacental cone in diabetic rats in vivo and in vitro.

Methods. Female Wistar rats were injected intraperitoneally with streptozotocin $(75 \mathrm{mg} / \mathrm{kg}$ body weight, $n=15)$, or with control buffer $(n=27) 3$ days before mating. On day 9 (day 1 = copulation plug) decidual swellings were weighed and the volume and mitotic index of the embryo and ectoplacental cone were estimated. Also, ectoplacental cones were cultured either in the presence of decidual cells from pseudopregnant diabetic rats or in high glucose concentration media. Cultures were evaluated by the daily outgrowth and by the proportion of giant cells and proliferating cells on day 5 .

Results. In diabetic rats on day 9, the weight of the decidual swellings and the mitotic index in the ectoplacental cone were lower compared with controls $(p<0.0001$ and $p<0.05$, respectively). In vitro, con- trol ectoplacental cones in the presence of decidual cells from diabetic rats showed a slight reduction in outgrowth on day 3 and 5 of culture. Outgrowth of diabetic ectoplacental cones in high glucose concentration medium was impaired on day $1(p<0.0005)$ compared with control ectoplacental cones in control medium, and on day 1 and 2 (both $p<0.005$ ) compared with control ectoplacental cones in high glucose concentration medium. In control medium, the outgrowth of diabetic ectoplacental cones was impaired on day $1(p<0.05)$, compared with control ectoplacental cones. Proliferation was stimulated in diabetic ectoplacental cone cultures.

Conclusion/interpretation. These data suggest that the outgrowth of diabetic ectoplacental cones is impaired by high glucose concentrations. [Diabetologia (2000) 43: 939-945]

Keywords Streptozotocin, decidua, ectoplacental cone, explant cultures, rats, trophoblast, placentation, cocultures, glucose, diabetes.
The altered metabolic environment in maternal diabetes during pregnancy interferes with normal fetal development. In humans, as well as in rodents, there is an increased incidence of congenital malforma-

Received: 22 December 1999 and in revised form: 22 March 2000

Corresponding author: S. Caluwaerts, Department of Obstetrics and Gynaecology, University Hospital Gasthuisberg, Herestraat 49, B-3000 Leuven, Belgium

Abbreviations: EPC, Ectoplacental cone; PCNA, proliferating cell nuclear antigen; DAB, 3,3'-diaminobenzidine tetrahydrochloride; TBS, Tris buffered saline. tions, such as non-closure of the neural folds and deformities of the heart chambers and the skeleton [1, $2]$, possibly induced by high glucose concentrations [3-5], low insulin concentrations [6,7] or ketone bodies [8]. Congenital malformations and fetal growth retardation in humans are found to be induced by the diabetic state as early as the late embryonic period (between the seventh and fourteenth week) [9]. In rodents experimental diabetes impairs, however, the development of the embryo even in the pre-implantation period [10-13]. By a precise metabolic control of diabetes during the early stage of pregnancy, the increased incidence of congenital malformations can be prevented $[14,15]$. 
Fetal development is highly dependent on an efficient nutrient supply through the placenta but also the decidua, the maternal supportive component of the placenta, plays a still ill-defined part and could be influenced by the diabetic milieu. The weight of the deciduomata of pseudopregnant diabetic rats on day 9 and 13 is lower than that of control rats [16, 17]. The placental weight of streptozotocin-induced diabetic rats at term is higher than that of control animals [18] and ultrastructural differences, such as the degeneration of the trophoblast cells in the labyrinthine zone, are reported at day 20 of gestation [19, $20]$. By contrast, the weight and nucleic acid content of placentas of diabetic rats on days 14 and 16 of gestation are statistically significantly lower and only from day 18 on, do the placental weight and nucleic acid content exceed that of control rats [21]. Moreover, the in vitro outgrowth of the trophoblast of blastocysts from diabetic rats is limited compared with the outgrowth of control blastocysts [22]. The latter study suggests that placental development in diabetic pregnancies is already impaired in the early stages.

Studies on the early placentation in diabetic rats are of extreme importance, as disturbances in the development of the preplacenta will affect proper functioning of the definitive placenta and inadequate transfer of compounds between mother and fetus can cause abnormalities in the developing fetus. Data on the outgrowth and proliferation of the postimplantation placental primordia of diabetic rats are, however, lacking. We examined the rat ectoplacental cone (EPC) tissue of diabetic and control rats in vivo, as well as the outgrowth of the EPC under different in vitro conditions. In diabetic rats on day 9 of pregnancy, we observed a reduction in the weight of decidual swellings and a reduction of the volume and mitotic index of the EPC. To examine the differential effect of impaired development of the decidua and high glucose concentrations, EPCs were dissected out and cultured in vitro, in the presence of decidual cells derived from diabetic or control rats or in high glucose medium.

\section{Materials and methods}

The entire protocol was reviewed and approved by the local ethics committee for animal procedures (Katholieke Universiteit, Leuven, Belgium).

Animals. Adult female Wistar rats between 100 and 120 days of age were used (Katholieke Universiteit Breeding Centre, Leuven, Belgium). Diabetes was induced 3 days before mating in 15 rats, using a single intraperitoneal injection of $75 \mathrm{mg} / \mathrm{kg}$ body weight streptozotocin (Upjohn, Puurs, Belgium), dissolved immediately before use in saline acidified to $\mathrm{pH} 4.5$ with citrate. On day 1 of gestation (the day of the copulation plug) glucose and insulin concentrations were measured in a tail vein blood sample. Plasma glucose concentration was determined using a glucose analyser (YSI 2300 Stat plus, Yellow
Springs Instruments, Yellow Springs, Ohio, USA). Insulin concentration was measured by radioimmunoassay with rat insulin as standard (Linco Research, St Charles, Mo., USA) [23]. Only rats with glucose concentrations exceeding $19.4 \mathrm{mmol} / \mathrm{l}$ and insulin concentrations less than $<25 \mu \mathrm{U} / \mathrm{ml}$ were included. It has been shown that glucose concentrations in severe diabetic rats remain consistently high over an extensive time [24]. Control rats $(n=27)$ were injected with $250 \mu$ of buffer 3 days before mating. Glucose concentrations on day 1 of gestation of these control rats were $7.1 \mathrm{mmol} / 1 \pm 0.72$. Rats had free access to standard rat laboratory chow (Trouw, Gent, Belgium) and to water. They were housed under controlled temperature and standard illumination cycles.

Processing and evaluation of implantation sites. On day 9 of pregnancy, six diabetic and eight control rats were killed by asphyxia with $\mathrm{CO}_{2}$, decidual swellings were peeled from the uterine horns and weighed. From every rat two decidual swellings were processed for histological evaluation. Decidual capsules were fixed in phosphate-buffered $4 \%$ paraformaldehyde ( $24 \mathrm{~h})$, subsequently washed in phosphate buffer-sucrose $\left(24 \mathrm{~h}, 4^{\circ} \mathrm{C}\right)$ and finally stored in ethanol $70 \%$ before embedding in paraffin. Step-serial sections of paraffin embedded decidual swellings were cut for cytokeratin immunostaining.

The total volume of the embryonic tissues and the ectoplacental cone was calculated by summing the surface areas of the different structures on adjacent sections, as measured using the Vidas automatic image analyser system (Kontron, Munich, Germany). The ectoplacental cone is defined as a mass of cytokeratin-positive trophoblastic cells, overlying the characteristically layered embryonic (i.e. endodermal and epidermal) structures. Mitotic indices were determined in three randomly chosen sections. For this purpose, the number of mitotic figures in the embryo and EPC were counted and expressed as a percentage of all cells in the embryo or EPC. The total number of cells counted range from 100-250 in the embryo and from $50-150$ in the EPC.

Processing and evaluation of ectoplacental cones cultures. Ectoplacental cones (EPCs) from diabetic $(n=9)$ and control rats $(n=9)$ on day 9 of pregnancy were dissected out under sterile conditions and transferred to an eight-well Nunc LabTek Chamber Slide (Vel, Haasrode, Belgium). Control culture medium was Dulbecco's modified Eagle medium (DMEM) supplemented with $10 \%$ fetal calf serum (FCS), $0.6 \%$ L-glutamine, $1 \%$ of a penicillin streptomycin solution and $0.1 \%$ gentamycin (all from Gibco, Merelbeke, Belgium). In some experiments, control medium was supplemented with $\mathrm{D}(+)$-glucose (Merck, Rahway, N.J., USA) to mimic the intra-uterine diabetic milieu (high glucose concentration medium). Final glucose concentrations are then $16.5 \mathrm{mmol} / \mathrm{l}$ glucose and the osmolarity of both control and high glucose concentration medium was $280 \mathrm{mOsm} / \mathrm{l}$.

Cultures were kept in the incubator at $37^{\circ} \mathrm{C}$, in an atmosphere of $5 \% \mathrm{CO}_{2}$ in air for 5 days. At the end of the experiment, the cultures were fixed during $90 \mathrm{~s}$ in a 50:50 paraformaldehyde ( $4 \%)$ : ethanol (100\%) mixture and stored in absolute ethanol at $4{ }^{\circ} \mathrm{C}$ until staining. Ectoplacental cones were photographed daily using an inverted phase-contrast microscope Leitz Fluovert (Leitz, Wetzlar, Germany). The surface area of the EPCs was measured on the photographs using the Vidas system. The percentage increase in outgrowth was defined as the surface area on day $\mathrm{X}$ divided by the surface area on day $(\mathrm{X}-1) \times 100$. This ratio reflects the percentage increase compared with the previous day; no growth is indicated by $100 \%$. The total surface area outgrowth was defined as the surface area on day 5 divided by the surface area on day $0(\times 100)$. 
The number of trophoblast giant cells was estimated using the Vidas system. The nuclei with an absolute area size greater than $35 \mu^{2}$ were counted and divided by the total surface area outgrowth to obtain the differentiation index. This index reflects the differentiation of the cultures in a specific experimental condition.

After immunohistochemical staining for proliferating cell nuclear antigen (PCNA), the proliferation rate was assessed as the percentage of PCNA-positive cells.

Collection of decidual cells. Vaginal smears were taken daily for 2 weeks to determine the oestrous cycle of the rats. We injected 10 rats with either a single intraperitoneal dose of $75 \mathrm{mg} / \mathrm{kg}$ streptozotocin or with control buffer 3 days before the induction of pseudopregnancy. Glucose and insulin concentrations were measured on the first day of pseudopregnancy. Pseudopregnancy was induced by cervical stimulation on the evening before and the morning after oestrus ( = day 1 of pseudopregnancy). On day 5 of pseudopregnancy, rats were anaesthetised using a mixture of Ketalar $(50 \mathrm{mg} / \mathrm{kg}$, ParkeDavis, Zaventem, Belgium), Rompun (10 mg/kg, Bayer, Brussels, Belgium) and Atropin Sulfas $(0.25 \mathrm{mg} / \mathrm{kg}$, Federa). In both uterine horns, $0.05 \mathrm{ml}$ mineral oil was injected for the induction of decidua development. On day 8 of pseudopregnancy, rats were killed by asphyxia, deciduomata were collected, minced and enzymatically dissociated with collagenase $(50 \mathrm{U} /$ $\mathrm{ml}$, Sigma Chemical, Bornem, Belgium), dispase $(2.4 \mathrm{U} / \mathrm{ml}$, Boehringer, Mannheim, Germany) and deoxyribonuclease (200 U/ml, Boehringer) in DMEM (40 min at $\left.37^{\circ} \mathrm{C}\right)$. Cells were collected by centrifugation over Ficoll-Paque (Pharmacia Biotech, Uppsala, Sweden) and seeded at concentrations of 300000 cells $/ \mathrm{ml}$. After $24 \mathrm{~h}$ in the incubator in an atmosphere of $5 \% \mathrm{CO}_{2}$ in $95 \%$ air, cultures were rinsed and EPCs from control rats $(n=10)$ were added. Ectoplacental cones were photographed on day 0 and on day 5 of culture, to measure the total surface area outgrowth.

\section{Immunohistochemistry}

Paraffin sections stained for cytokeratin. After digestion with pepsin (P7012, Sigma Chemical) in $\mathrm{HCl}$, slides were subsequently treated with a mixture of $\mathrm{H}_{2} \mathrm{O}_{2}$ and $\mathrm{NaN}_{3}$ in methanol (30 $\mathrm{min})$ and with a $2 \%$ BSA solution $(15 \mathrm{~min})$. Slides were then incubated with a mouse anti-cytokeratin $(1 / 400$, Clone MNF 116, Dako, Glostrup, Denmark) for $2 \mathrm{~h}$ at room temperature. The secondary antibody was a peroxidase conjugated goat anti-mouse IgG (1/100, $30 \mathrm{~min}$, Jackson ImmunoResearch Laboratories, West Grove, Pa., USA), supplemented with normal rat serum $1 / 25$. Peroxidase activity was detected using 3,3'-diaminobenzidine tetrahydrochloride (DAB, Sigma Chemical) and slides were counterstained with Harris' haematoxylin (Merck). Slides were coded and evaluated without knowledge of treatment.

Ectoplacental cone cultures stained for PCNA. Immunohistochemical staining for the proliferation marker PCNA was done on the Lab-Tek slides.

Slides were treated for 30 min with a mixture of $\mathrm{H}_{2} \mathrm{O}_{2} 0.5 \%$ and $\mathrm{NaN}_{3} 0.1 \%$ in TRIS-buffered saline (TBS) and were preincubated during 15 min with normal goat serum (Dako) 1/30 and $0.5 \%$ Tween 80 (Merck). The primary antibody was a monoclonal mouse anti-rat PCNA $(1 / 100,2 \mathrm{~h}$, Clone PC10, Dako), followed by 15 min blocking with normal goat serum and the secondary antibody, a peroxidase conjugated goat anti-mouse $\operatorname{IgG}(1 / 100,30 \mathrm{~min}$, Jackson ImmunoResearch
Laboratories). We used TBS as a diluent. Peroxidase activity was shown using a standard DAB solution for $7 \mathrm{~min}$. Cultures were counterstained with Mayer's haematoxylin (Merck) and mounted with glycerine jelly. Slides were coded and evaluated without knowledge of treatment.

Cocultures stained for desmin. The presence of desmin was evaluated as a marker for decidual cells [25].

After 15 min of blocking with $2 \%$ BSA, supplemented with Tween $80(0.5 \%)$, Lab-Tek slides were incubated with mouseanti-desmin (1/800, Clone D33, Dako) for $30 \mathrm{~min}$. Slides were treated with a mixture of $\mathrm{H}_{2} \mathrm{O}_{2}$ and $\mathrm{NaN}_{3}$ for $30 \mathrm{~min}$. After blocking, slides were incubated with a peroxidase conjugated goat anti-mouse (1/100, Jackson ImmunoResearch Laboratories), supplemented with normal rat serum 1/25. Detection with 3-amino-9-ethylcarbazole (10 min, Sigma Chemical) resulted in cells positive for desmin being stained red. Slides were counterstained with Mayer's haematoxylin and mounted with glycerine jelly.

Statistics. Results of treatments were compared using an unpaired $t$ test, with $p$ less than 0.05 considered to be significantly different. The significant differences in the outgrowth of the EPCs in vitro were detected using an unpaired $t$ test with Welch correction. Data were expressed as means \pm SEM.

\section{Results}

Implantation sites. In diabetic rats, the mean weight of the decidual swellings $(12.2 \pm 0.94 \mathrm{mg}, n=61)$ was lower $(p<0.0001)$ than in control rats $(24.4 \pm$ $0.78 \mathrm{mg}, n=93)$. The total volume of the embryonic and ectoplacental cone tissue in diabetic rats $\left(3.35 \pm 0.44 \times 10^{6} \mu \mathrm{m}^{3}\right.$ and $2.63 \pm 0.41 \times 10^{6} \mu \mathrm{m}^{3}$, respectively; $n=9$ ) was not significantly different from controls $\left(3.45 \pm 0.56 \times 10^{6} \mu \mathrm{m}^{3}\right.$ and $3.14 \pm 0.50 \times 10^{6}$, respectively; $n=13$, Fig. 1 ). The mitotic index (Fig.1) in the embryonic tissue of diabetic rats $\left(56 \pm 8.9 \times 10^{-3}\right)$ was slightly lower than in the controls $\left(73 \pm 5.8 \times 10^{-3}\right)$. In the EPC, the mitotic index in diabetic rats was $34 \pm 6.9 \times 10^{-3}$ and lower $(p<0.05)$ than in control rats $\left(57 \pm 5.8 \times 10^{-3}\right)$.

Effect of decidual cells on EPC outgrowth in vitro. To evaluate a possible effect of diabetic decidua on the outgrowth of the ectoplacental cone, decidual cells from pseudopregnant diabetic and control rats were cocultured with EPCs from control rats under normal glucose concentrations. After 3 days in culture, the total surface area outgrowth of the EPC in the presence of control and diabetic decidual cells was $2.21 \pm 0.42 \times 10^{5} \mu \mathrm{m}^{2}(n=12)$ and $1.61 \pm 0.25 \times 10^{5}$ $\mu \mathrm{m}^{2}(n=18)$ respectively. After 5 days in culture, this number was $3.48 \pm 1 \times 10^{5} \mu \mathrm{m}^{2} \quad(n=9)$ and $3.42 \pm 0.57 \times 10^{5} \mu \mathrm{m}^{2}(n=12)$, respectively. No significant differences could be shown.

Effect of glucose concentrations on EPC outgrowth in vitro. The data on these experiments are summarised in Table 1. 

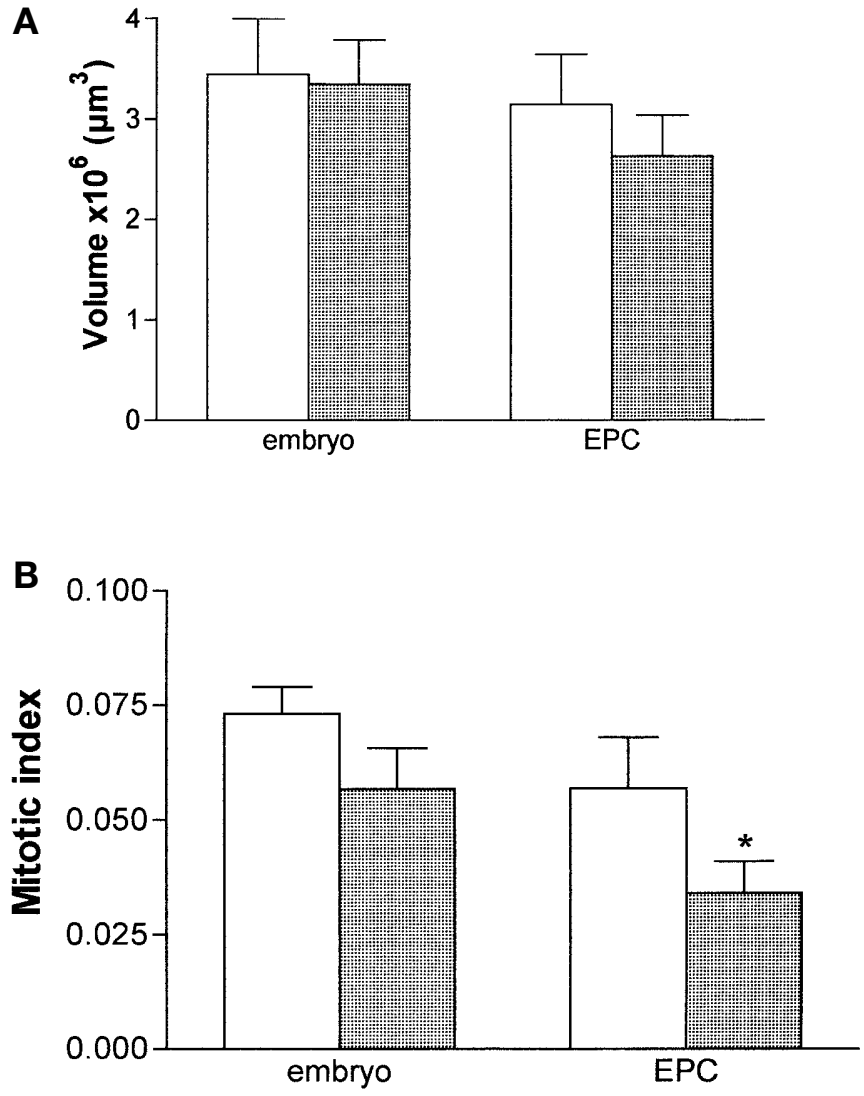

Fig. 1. A Volume of embryo and ectoplacental cone of control $(\square, n=8)$ and diabetic rats (圆, $n=6)$ on day 9 of pregnancy. Values are given as means \pm SEM. B Mitotic index of embryo

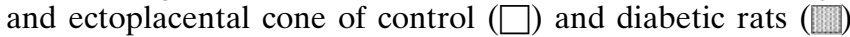
on day 9 of pregnancy. Values are given as means \pm SEM. $* p<0.05$ vs values in control rats

Outgrowth of EPCs from control rats in control medium compared with outgrowth of EPCs from diabetic rats in high glucose concentration medium. On day 1, the percentage increase in surface area of EPCs from control rats was higher than the outgrowth of EPCs from diabetic rats $(p<0.0005$, Fig. 2$)$. Outgrowth of the EPCs from diabetic rats was still im- paired during the second day, although no statistically significant differences could be measured. Outgrowth decreased from the third day on and was approximately equal for EPCs from both diabetic and control rats during the last days of culture (NS).

The total increase in surface area of EPC from control rats in control medium was $530.05 \pm 73.91 \%$, whereas the outgrowth of EPCs from diabetic rats in high glucose concentration medium was $343.33 \pm$ $71.9 \%$ (NS). The differentiation index was $1.3 \pm 0.28$ for EPCs from control rats and $0.94 \pm 0.19$ for EPCs from diabetic rats. The mean percentage of cells positive for PCNA for both control and diabetic rats was $41.6 \pm 2.11 \%$ and $60.46 \pm 2.88 \%$, respectively $(p=0.0005$, Fig. 3$)$.

These results strongly suggest that the outgrowth of EPCs of diabetic rats is impaired in medium mimicking the diabetic intra-uterine milieu. Proliferation of the cells seems, however, to be stimulated.

Outgrowth of EPCs from control and diabetic rats in high glucose concentration medium. To evaluate to what extent the impaired outgrowth of EPCs from diabetic rats is due to the negative effect of high glucose concentrations, both EPCs from diabetic and control rats were cultured in high glucose concentration medium and outgrowth was compared (Fig.2). After $24 \mathrm{~h}$ in culture, the increase in surface area of EPCs from control rats in high glucose concentration medium was higher than the outgrowth of EPCs from diabetic rats $(p<0.005)$. This difference remains after 2 days $(p<0.005)$. Growth rates during the last 3 days were more or less the same in both conditions. The total increase of the surface area over 5 days was $671.3 \pm 94.76 \%$ for EPCs from control rats in high glucose concentration medium and $343.33 \pm 71.09 \%$ for EPCs from diabetic rats $(p<0.05)$. The differentiation index was $1.20 \pm 0.32$ for EPCs from control rats and $0.94 \pm 0.19$ for EPCs from diabetic rats in high glucose concentration medium (NS). Proliferation was $52.11 \pm 2.54 \%$ for EPCs from control rats and $60.46 \pm 2.88 \%$ for EPCs

Table 1. Daily outgrowth of ectoplacental cones (EPCs) from control and diabetic rats on day 9 of pregnancy over 5 days in culture

\begin{tabular}{lllll}
\hline & $\begin{array}{l}\text { Control EPC } \\
\text { in control medium }(\%) \\
(n=20)\end{array}$ & $\begin{array}{l}\text { Control EPC } \\
\text { in high glucose } \\
\text { medium }(\%) \\
(n=21)\end{array}$ & $\begin{array}{l}\text { Diabetic EPC } \\
\text { in control medium }(\%) \\
(n=25)\end{array}$ & $\begin{array}{l}\text { Diabetic EPC } \\
\text { in high glucose } \\
\text { medium }(\%) \\
(n=16)\end{array}$ \\
\hline Day 1 & $175.73( \pm 14.44)$ & $198.22( \pm 22.97)$ & $126.42( \pm 12.16)^{\mathrm{a}, \mathrm{b}}$ & $104.95( \pm 7.91)^{\mathrm{c}, \mathrm{d}}$ \\
Day 2 & $247.59( \pm 21.64)$ & $329.62( \pm 29.28)^{\mathrm{e}}$ & $296.54( \pm 25.4)$ & $189.59( \pm 35.58)^{\mathrm{f}, \mathrm{g}}$ \\
Day 3 & $134.05( \pm 10.8)$ & $120.1( \pm 10.32)$ & $146.27( \pm 9.67)$ & $141.47( \pm 11.09)$ \\
Day 4 & $109.63( \pm 6.52)$ & $110.41( \pm 6.31)$ & $116.52( \pm 7.77)$ & $125.14( \pm 10.65)$ \\
Day 5 & $94.96( \pm 5.75)$ & $91.37( \pm 5.1)$ & $103.86( \pm 5.66)$ & $121.05( \pm 21.26)$ \\
\hline
\end{tabular}

Values are given as means \pm SEM. The number of animals is given within parentheses. High glucose medium $=$ high glucose concentration medium. ${ }^{\mathrm{a}} p<0.05$ vs EPC (control)/control medium, ${ }^{\mathrm{b}} p<0.01$ vs EPC (control)/high glucose medium,
${ }^{\mathrm{c}} p<0.0005$ vs EPC (control)/control medium, ${ }^{\mathrm{d}} p<0.005 \mathrm{vs}$ EPC (control)/high glucose medium, ${ }^{\mathrm{e}} p<0.05$ vs EPC (control)/control medium, ${ }^{\mathrm{f}} p<0.005$ vs EPC (control)/high glucose medium, ${ }^{\mathrm{g}} p<0.05$ vs EPC (diabetic)/control medium 

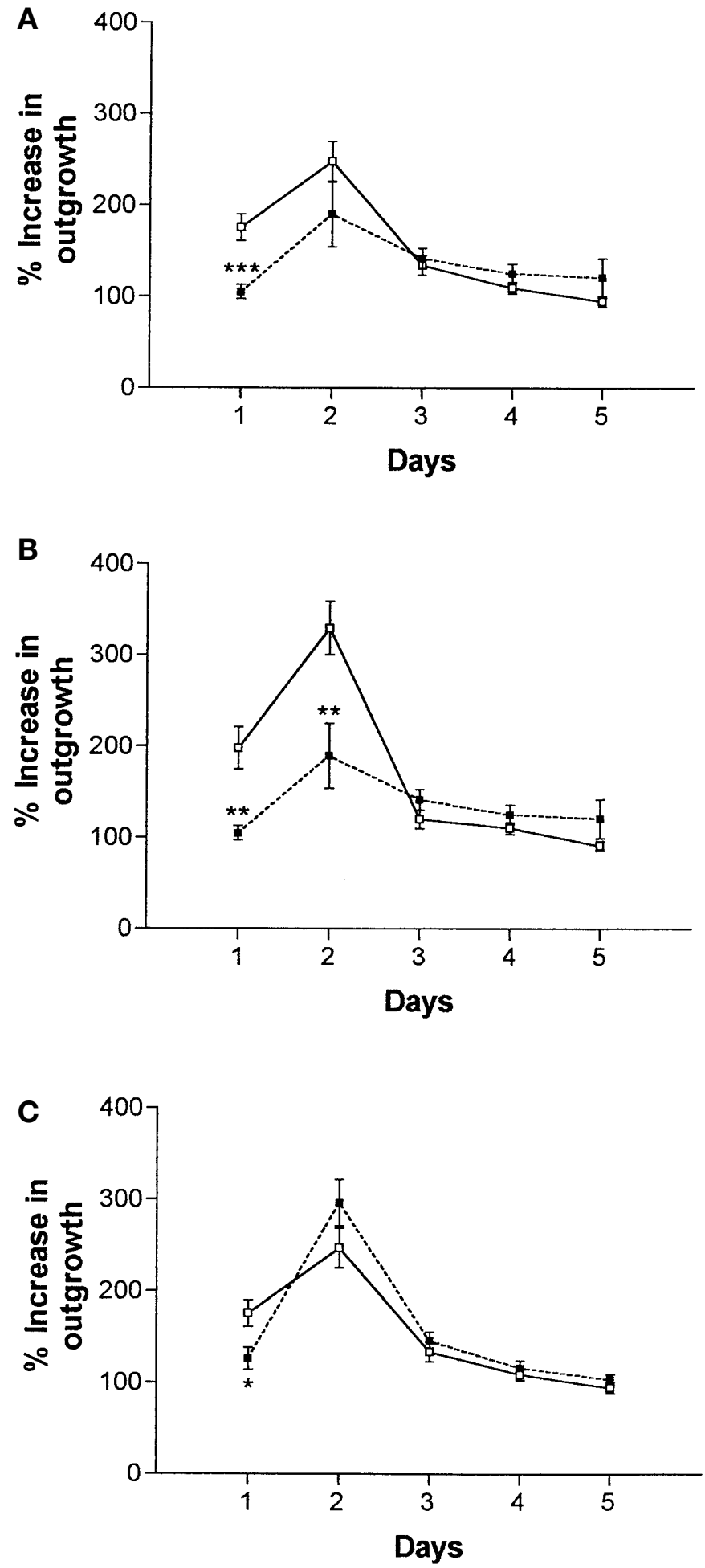

Fig. 2 A-C. Daily outgrowth of ectoplacental cones (EPCs) from control $(\square, n=9)$ and diabetic rats $(\square, n=9)$ on day 9 of pregnancy over 5 days in culture. Outgrowth is expressed as a percentage of the surface area on day $\mathrm{X}$, divided by the surface area on day (X-1). Values are given as means \pm SEM. A EPCs from control rats in control medium vs EPCs from diabetic rats in high glucose concentration medium, $* * * p<0.0005$. B EPCs from control and diabetic rats in high glucose concentration medium, $* * p<0.005$. C EPCs from control and diabetic rats in control medium, ${ }^{*} p<0.05$

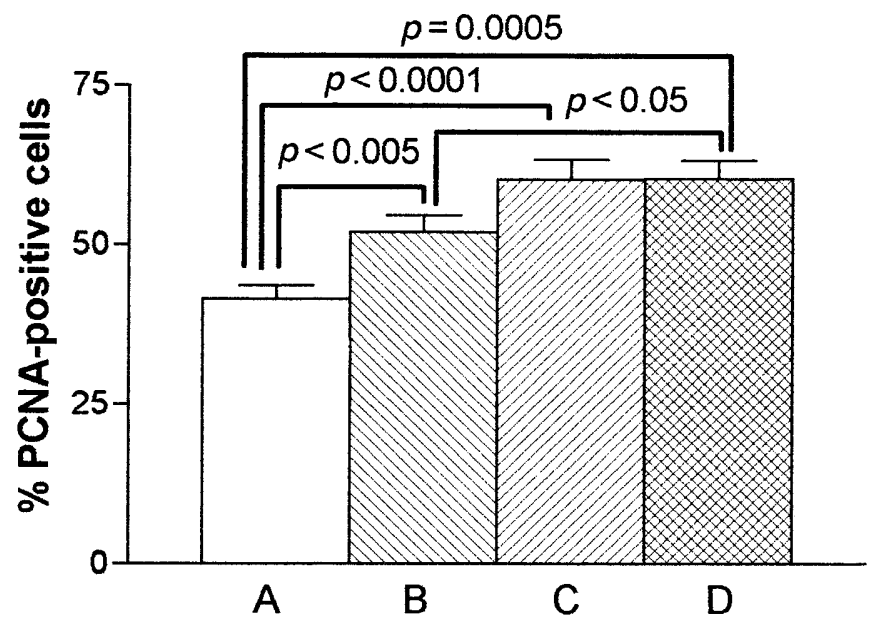

Fig.3. Percentage PCNA-positive cells in ectoplacental cones from control rats $(n=9)$ in control medium (A) and in high glucose concentration medium (B), and from diabetic rats $(n=9)$ in control medium (C) and in high glucose concentration medium (D) after 5 days in culture. Values are given as means \pm SEM

from diabetic rats in high glucose concentration medium $(p<0.05$, Fig. 3$)$.

From these experiments we conclude that glucose alone gives no satisfactory explanation for the impaired outgrowth capacity of EPCs from diabetic rats in the presence of high glucose concentrations. Glucose does, however, stimulate the proliferation and outgrowth of EPCs from control rats, (Table 1, $p<0.05$ on day 2 ).

Outgrowth of the EPCs from control and diabetic rats in control medium. To examine the effect of a normalised glycaemia on the outgrowth of EPCs from diabetic rats, EPCs from control and diabetic rats were cultured in control medium (Fig.2). During the first day, the percentage increase in surface area was higher in the EPCs from control rats than those from diabetic rats $(p<0.05)$. From the second day on, the increase in surface area outgrowth was equal in the EPCs from control and diabetic rats.

The total increase of the surface area over 5 days was $530.05 \pm 73.91 \%$ for EPCs from control rats and $578.17 \pm 80.77 \%$ for EPCs from diabetic animals. The differentiation index is $1.31 \pm 0.28$ and $1.47 \pm 0.3$ in control and diabetic rats, respectively in control medium. The estimated average percentage of PCNA positive nuclei was $41.59 \pm 2.11 \%$ in cultures of control rats and $60.26 \pm 3.17 \%$ in cultures of diabetic rats $(p<0.0001$, Fig. 3$)$.

These results show that outgrowth of the EPCs from diabetic rats is still impaired in normalised glucose concentrations although the difference in outgrowth capacity is smaller. 


\section{Discussion}

Previous reports have shown that in diabetic rats, the development of the decidua on day 9 and on day 13 of pseudopregnancy is impaired [16,17] which is confirmed by our results. This could be due to a reduced uterine blood flow [26] or a reduction in serum progesterone concentrations in diabetic pseudopregnant rats. Although the exact role of the decidua in the process of implantation and placentation is not clear, important functions could be the nourishment of the developing embryo and the control of trophoblast invasion. To examine the effect of the impaired decidual reaction in diabetic pregnancies on trophoblast outgrowth, we have studied the total volume and the mitotic index of the embryo and EPC in decidual swellings on day 9 of pregnancy. In diabetic pregnancies, the mitotic index of the EPC is reduced compared with control pregnancies. Therefore we conclude that an impaired decidual cell response has an indirect effect on trophoblast development.

Pure trophoblast can be obtained from the EPC of mice on day 7 to 8 of pregnancy [27]. Ectoplacental cones can therefore be considered as the placental primordia and were used in an in vitro system to examine trophoblast development. The effect of decidual cells of diabetic and control pseudopregnant rats on EPC outgrowth was studied in coculture. Although the outgrowth of the EPC of control rats in the presence of diabetic decidual cells was reduced on day 3 and day 5, no statistically significant differences were measurable. This could be due to a failure of cultured decidual cells to maintain their differentiated state, although the decidual cell marker desmin [25] was expressed throughout the period of culture.

Increased glucose concentrations have been shown to play a major part in the impaired development of the embryos of diabetic pregnant rats [28]. These results are compatible with the results of our study in which we have shown a difference in trophoblast outgrowth between control and diabetic rats. Ectoplacental cones were cultured in vitro in medium mimicking the glycaemic condition of the intra-uterine milieu to investigate its effect on trophoblast. When EPCs from control rats were cultured in control medium and EPCs from diabetic animals were cultured in high glucose concentration medium, the outgrowth of the EPCs of diabetic rats is impaired during the first two days in culture. An impaired outgrowth of the EPCs in culture can be caused by a decrease in proliferation, differentiation or spreading of the trophoblast cells. Based on the PCNA-staining, the proliferation of the EPCs from diabetic rats is higher than those of EPCs from control rats. These results seem, however, to conflict with the reduced mitotic index observed in the EPCs of diabetic rats in vivo. Both measures of cell proliferation have, however, their own limitations. Mitotic index evaluations, if not done in colchicine- treated animals, only provide information within a time-restricted window. Immunostaining for PCNA has, however, an inherent problem because this particular antigen can be retained for a prolonged period during the cell cycle. It is clear that, whatever the exact mechanism, statistically significant changes take place in EPC cell cycles in a high glucose concentration milieu. Along the same line it has been hypothesised by others that hyperglycaemia leads to a relative immaturity of rat placentas by providing a stimulus for continuous growth and cell division [29]. Our findings of a decreased differentiation index in diabetic rats are also in agreement with this hypothesis. The decreased outgrowth capacity of EPCs from diabetic rats could, however, be due to a decreased ability of adhering and spreading on the plastic surface. This could result in a different expression of adhesion molecules in the EPCs from diabetic rats from those from control rats. Spreading of the cells onto the plastic surface is also strongly influenced by the composition of the cell membranes; electrostatic potentials can stimulate or inhibit the binding of the cells onto the surface.

It has been shown that glucose has teratogenic effects on embryonic development [10-13]. Also, the impaired outgrowth of the EPC of diabetic rats could be specifically caused by the presence of high glucose concentrations. Therefore, we have cultured the EPCs from control animals in high glucose concentrations and compared them with the culture of EPCs from diabetic rats in glucose supplemented medium. High glucose concentrations stimulate, however, the outgrowth of EPCs from control rats on day 2 , which is due to an increase in cell proliferation in high glucose concentration medium compared with control medium. We can therefore conclude that glucose stimulates the outgrowth of EPCs from control rats but worsens the impaired outgrowth of EPCs from diabetic rats. Other factors such as reduced insulin concentrations are, however, very likely to play a part. Osmotic features cannot explain the impairment in outgrowth capacity, as the osmolarity in both control and high glucose media is equal.

We compared the outgrowth of EPCs from control rats with EPCs from diabetic rats, both in control medium. Outgrowth of the EPCs from diabetic rats was still impaired, although to a lesser extent and only during the first day. After $48 \mathrm{~h}$, outgrowth of the EPCs from diabetic rats is similar to that of control EPCs if glucose concentrations are normalised. Hence, the outgrowth of EPCs from diabetic rats in culture is impaired on day 1 , both in control medium and in high glucose concentration medium. The difference in outgrowth capacity between diabetic and control EPCs is, however, greater in high glucose concentration medium, which suggests that hyperglycaemia worsens the effect of diabetes. It is possible that the diabetic intra-uterine milieu causes an increased expression of glucose transporters in the embryo and 
trophoblast, leading to exposure of the cells to excessive glucose concentrations [30]. After $48 \mathrm{~h}$, the outgrowth of the diabetic EPCs in vitro is still impaired in high glucose concentration medium but normalised in control medium.

This study in the diabetic pregnant rat suggests an impairment in the early development of the trophoblast, even before anomalous organogenesis can be observed. Impairment in the development of trophoblast will lead to an inadequate nutrient transfer through the placenta, which can possibly cause disturbances in fetal growth and development. Whether or not this is true in human pregnancy needs to be clarified. It has, however, been shown that metabolic derangements during early human pregnancy increase the occurrence of congenital malformations [15]. This study emphasises that diabetes in human pregnancy needs to be controlled, even in the earliest stages.

The outgrowth of the EPCs from diabetic rats is impaired compared with that of control rats. On day 9 of pregnancy, this impaired outgrowth is partly due to high concentrations of glucose. Normalising the blood glucose concentrations in diabetic rats could therefore normalise trophoblast outgrowth after $48 \mathrm{~h}$.

Acknowledgements. This study was supported by the Fonds voor Wetenschappelijk Onderzoek - Vlaanderen (Fund for Scientific Research-Flanders, Belgium, FWO) (1.5.649.98).

\section{References}

1. Eriksson U (1984) Congenital malformations in diabetic animal models - a review. Diabetes Res 1: 57-66

2. Deuchar E (1977) Embryonic malformations in rats, resulting from maternal diabetes: preliminary observations. J Embryol Exp Morphol 41: 93-99

3. Cockroft D (1984) Abnormalities induced in cultured rat embryos by hyperglycemia. Br J Exp Pathol 65: 625-636

4. Garnham E, Beck F, Clarke C, Stanisstreet M (1983) Effects of glucose on rat embryo in culture. Diabetologia 25: 291-295

5. Cockroft D, Coppola P (1977) Teratogenic Effects of Excess Glucose on head-fold rat embryos in culture. Teratology 16: 141-146

6. Beebe L, Kaye P (1991) Maternal diabetes and retarded preimplantation development of mice. Diabetes 40: 457-461

7. Travers J, Pratten M, Beck F (1989) Effects of low insulin levels on rat embryonic growth and development. Diabetes 38: 773-778

8. Zusman I, Yaffe P, Ornoy A (1987) Effects of metabolic factors in the diabetic state on the in vitro development of preimplantation mouse embryos. Teratology 35: 77-85

9. Pedersen J, Molsted-Pedersen L (1981) Early fetal growth delay detected by ultrasound marks increased risk of congenital malformation in diabetic pregnancy. BMJ 283: 269-271

10. Diamond M, Moley K, Pellicer A, Vaughn W, DeCherney A (1989) Effects of streptozotocin- and alloxan-induced diabetes mellitus on mouse follicular and early embryo development. J Reprod Fertil 86: 1-10
11. Pampfer S, De Hertogh R, Vanderheyden I, Michiels B, Vercheval M (1990) Decreased inner cell mass proportion in blastocysts from diabetic rats. Diabetes 39: 471-476

12. Vercheval M, De Hertogh R, Pampfer S et al. (1990) Experimental diabetes impairs rat embryo development during the preimplantation period. Diabetologia 33: 187-191

13. De Hertogh R, Vanderheyden I, Pampfer S, Robin D, Dufrasne E, Delcourt J (1991) Stimulatory and inhibitory effects of glucose and insulin on rat blastocyst development in vitro. Diabetes 40: 641-647

14. Baker L, Egler J, Klein S, Goldman A (1981) Meticulous control of diabetes during organogenesis prevents congenital lumbosacral defects in rats. Diabetes 30: 955-959

15. Fuhrmann K, Reiher H, Semmler K, Fisher F, Fisher M, Glöckner E (1983) Prevention of congenital malformations in infants of insulin-dependent diabetic mothers. Diabetes Care 6: 219-223

16. Garris D (1988) Effects of diabetes on uterine condition, decidualization, vascularization, and corpus luteum function in the pseudopregnant rat. Endocrinology 122: $665-672$

17. Norambuena J, Pijnenborg R, Van Assche F, Brosens I (1984) Decidual changes in the endometrium and morphological adaptation of the associated supplying arteries in the normal and diabetic pseudopregnant rat. Placenta 5: 249-260

18. Aerts L, Holemans K, Van Assche F (1990) Maternal diabetes during pregnancy: consequences for the offspring. Diabetes Metab Rev 6: 147-167

19. Padmanabhan R, al-Zuhair A (1990) Ultrastructural studies on the placentae of streptozotocin induced maternal diabetes in the rat. Z Mikrosk Anat Forsch 104: 212-230

20. Prager R, Abramovici A, Liban E, Laron Z (1974) Histopathological changes in the placenta of streptozotocin induced diabetic rats. Diabetologia 10: 89-91

21. Robinson J, Canavan J, El Haj A, Goldspink D (1988) Maternal diabetes in rats 1 . Effects on placental growth and protein turnover. Diabetes 37: 1665-1670

22. Pampfer S, Wuu Y, Vanderheyden I, De Hertogh R (1994) In vitro study of the carry-over effect associated with early embryopathy in the rat. Diabetologia 37: 855-862

23. Yalow R, Berson S (1960) Immunoassay of endogenous plasma insulin in man. J Clin Invest 39: 1157-1175

24. Holemans K, Aerts L, Van Assche F (1991) Evidence for an insulin resistance in the adult offspring of pregnant streptozotocin-diabetic rats. Diabetologia 34: 81-85

25. Glasser S, Lampelo S, Munir M, Julian J (1987) Expression of desmin, laminin and fibronectin during in situ differentiation (decidualization) of rat uterine stromal cells. Differentiation 35: 132-142

26. Palacin M, Lasuncion M, Martin A, Herrera E (1985) Decreased uterine blood flow in the diabetic pregnant rat does not modify the augmented glucose transfer to the fetus. Biol Neonate 48: 197-203

27. Rossant J, Croy A (1985) Genetic identification of tissue of origin of cellular populations within the mouse placenta. $\mathbf{J}$ Embryol Exp Morphol 86: 177-189

28. Diamond M, Pettway Z, Logan J, Moley K, Vaughn W, DeCherney A (1991) Dose-response effects of glucose, insulin, and glucagon on mouse pre-embryo development. Metabolism 40: 566-570

29. Gewolb I, Merdian W, Warshaw J, Enders A (1986) Fine structural abnormalities of the placenta in diabetic rats. Diabetes 35: 1254-1261

30. Boileau P, Mrejen C, Girard J, Hauguel-de Mouzon S (1995) Overexpression of GLUT3 placental glucose transporter in diabetic rats. J Clin Invest 96: 309-317 